\title{
A New Branch of the Pure Algebra: BCL-Algebras
}

\author{
Yonghong Liu \\ School of Automation, Wuhan University of Technology, Wuhan, China \\ E-mail:hylinin@163.com \\ Received May 18, 2011; revised June 23, 2011; accepted July 5, 2011
}

\begin{abstract}
The BCK/BCI/BCH-algebras finds general algebra system than Boolean algebras system. This paper presents a novel class of algebras of type $(2,0)$ called BCL-algebras. We found the BCL-algebras to be more extensive class than BCK/BCI/BCH-algebras in the abstract algebra. The BCL-algebras as a class of logical algebras are the algebraic formulations of the set difference together with its properties in set theory and the propositional calculus in logical systems. It is important that the BCL-algebras play an independent role in the axiom algebra system.
\end{abstract}

Keywords: Logic Algebra, BCK-Algebra, BCI-Algebra, BCH-Algebra, BCL-Algebra

\section{Introduction}

In [1,2], the BCK-algebras and BCI-algebras are abbreviated to two B-algebras. The former was raised in 1966 Y. Imai and $\mathrm{K}$. Iséki, and the latter was primitives in the same year due to K. Iséki [3]. In 1983, Q. P. Hu and X. $\mathrm{Li}[4,5]$ defined a class of algebras of type $(2,0)$ called $\mathrm{BCH}$-algebras base on BCK-algebras and $\mathrm{BCI}$-algebras. In this paper we present the BCL-algebras, namely, L-algebras of type $(2,0)$.

To begin with, let's examine BCI-algebras and BCKalgebras that we have all observed. In fact, BCK-algebras are a special class of BCI-algebras; we have the following nice results.

Definition 1.1. [2] An algebra $(X ; *, 0)$ of type $(2,0)$ is called a BCI-algebra if it satisfies the following conditions: for any $x, y, z \in X$,

1) BCI-1: $((x * y) *(x * z)) *(z * y)=0$;

2) BCI-2: $(x *(x * y)) * y=0$;

3) BCI-3: $x^{*} x=0$;

4) BCI-4: $x^{*} y=0$ and $y^{*} x=0$ imply $x=y$;

5) BCI-5: $x^{*} 0=0$ imply $x=0$.

Theorem 1.1. [3] Given a BCI-algebra $X$, then the following identity holds: for any $x, y, z \in X$,

$$
\left(x^{*} y\right) * z=\left(x^{*} z\right) * y=0
$$

Definition 1.2. [6] Given a BCI-algebra $X$, if it satisfies the condition

BCK-4: $0 * x=0$ for all $x \in X$ (i.e., every element $x \in X$ is positive), we call it the BCK-algebra.

Definition 1.3. [4] An algebra $(X ; *, 0)$ of type $(2,0)$ is called a $\mathrm{BCH}$-algebra if it satisfies the following conditions: for any $x, y, z \in X$,

1) BCH-1: $\quad x^{*} x=0$;

2) BCH-2: $\quad x * y=0$ and $y * x=0$ imply $x=y$;

3) BCH-3: $(x * y) * z=\left(x^{*} z\right) * y$.

\section{BCL-Algebras}

There are several axiom systems for BCL-algebras as shown in the following.

Definition 2.1. An algebra $\left(X ;^{*}, 0\right)$ of type $(2,0)$ is said to be a BCL-algebra if and only if for any $x, y, z$ in $X$, the following conditions:

1) BCL-1: $\quad x^{*} x=0$;

2) BCL-2: $x^{*} y=0$ and $y * x=0$ imply $x=y$;

3) BCL-3: $(((x * y) * z) *((x * z) * y)) *((z * y) * x)=0$.

Such set $X$ in Definition 2.1 is called the underlying set of a BCL-algebra $(X ; *, 0)$.

Definition 2.2. Let $(X ; *, 0)$ is a BCL-algebra. A binary relation $\leq$ on $X$ by which $x \leq y$ if and only if $x^{*} y=0$ for any $x, y, z \in X$, we call the BCL-ordering $\leq$ is a partial ordering on $X$.

Definition 2.3. Let $x \leq y$ if and only if $x^{*} y=0$, the Definition 2.1 can then be written as

1) BCL-1*: $x \leq x$;

2) BCL-2*: $x \leq y$ and $y \leq x$ imply $x=y$;

3) BCL-3*: $((x * y) * z) *((x * z) * y) \leq(z * y) * x$.

Definition 2.4. From the class of all BCL-algebra, we denote by BCL.

Theorem 2.1. 1) Any a BCK-algebra is a BCL-algebra; 
2) Any a BCI-algebra is a BCL-algebra;

3) Any a BCH-algebra is a BCL-algebra.

Proof: For Theorem 2.1 1), 2) and 3), notes how the basic fact- BCK-algebraic class $\subset$ BCI-algebraic class $\subseteq \mathrm{BCH}$-algebraic class, we only need to prove the following result.

Let $\left(X ;^{*}, 0\right)$ be a $\mathrm{BCH}$-algebra, suppose that $x, y$, $z \in X, 0 * y=y$ and $x^{*} y=z$, using BCH-1 and BCH3 then

$$
\begin{aligned}
& (((x * y) * z) *((x * z) * y)) *((z * y) * x) \\
& =((z * z) *(x * y) * z) *((z * x) * y) \\
& =(0 *(z * z)) *(((x * y) * x) * y) \\
& =(0 * 0) *(((x * x) * y) * y) \\
& =0 *((x * y) * y) \\
& =0 *(y * y) \\
& =0 * 0=0
\end{aligned}
$$

Therefore, $(X ; *, 0)$ be a BCL-algebra.

Theorem 2.2. Let $\left(X ;^{*}, 0\right)$ be a BCL-algebra, if

$$
(x * y) * z=(x * z) * y \text { for any } \mathrm{x}, \mathrm{y}, \quad \mathrm{z} \in X
$$

Then the BCL-algebra is a BCH-algebra.

Proof: Let $(X ; *, 0)$ be a $\mathrm{BCH}$-algebra, suppose that $x, y, \quad z \in X, 0^{*} y=y$ and $x^{*} y=z$, using BCH-1 and BCH-3, by Theorem 2.1(3), we have $(x * y) * z=0$.

Similarly, suppose that $x, y, z \in X \quad 0^{*} z=z$ and $x * z=y$, using BCH-1 and BCH-3. We then have

$$
\begin{aligned}
& \left(\left(\left(x^{*} z\right) * y\right) *((x * y) * z)\right) *((y * z) * x) \\
& =((y * y) *(x * z) * y) *((y * x) * z) \\
& =(0 *(y * y)) *(((x * z) * x) * z) \\
& =(0 * 0) *\left(\left(\left(x^{*} x\right) * z\right) * z\right) \\
& =0 *((x * z) * z) \\
& =0 *(z * z) \\
& =0 * 0=0
\end{aligned}
$$

Hence $\left(x^{*} z\right) * y=0$. Combining this with equation is $\left(x^{*} y\right) * z=0$, we obtain equation (3). Finally, the Theorem 2.2 is proved.

As this Theorem 2.1 and 2.2 indicate, we summarize these results in the following contains.

Corollary 2.1. BCK-algebraic class $\subset$ BCI-algebraic class $\subset \mathrm{BCH}$-algebraic class $\subseteq$ BCL-algebraic class $\subset A(2,0)$, where $A(2,0)$ is algebraic class of type $(2,0)$.

Theorem 2.3. Let $(X ; *, 0)$ be a BCL-algebra. Then the following hold: for any $x, y, z \in X$,

1) BCL-3*: $((x * y) * z) *((x * z) * y) \leq(z * y) * x$;
2) $x^{*} y=0$ if and only if $x \leq y$.

Proof: Assume that $(X ; *, 0)$ is a BCL-algebra, then the BCL-ordering $\leq$ is a partial ordering on $X$. By definition of $\leq,(2)$ is valid. Also, BCL-3 and (2) imply (1).

Conversely, assume that $\leq$ is a partial ordering on $X$, and satisfying (1) and (2). Also, by the reflexivity of $\leq$, we see that $x \leq x$, then (2) implies $x^{*} x=0$.

Moreover, if $x^{*} y=0$ and $y^{*} x=0$, then $x \leq y$ and $y \leq x$ by (2), and so the anti-symmetry of $\leq$ gives $x=y$.

Therefore $(X ; *, 0)$ is a BCL-algebra.

Definition 2.5. If a BCL-algebra $(X ; *, 0)$ is not $\mathrm{BCK} / \mathrm{BCI} / \mathrm{BCH}$-algebra, it is called proper $\mathrm{BCL}$-algebra.

Theorem 2.4. There is an algebra $(X ; *, 0)$ of type $(2,0)$ which proper BCL-algebra is exists.

Proof: The proof of this Theorem 2.4 is not difficult and uses only example. Let $X=\{0,1,2,3\}$. Define an operation * on $\mathrm{X}$, we find

\begin{tabular}{l|llll}
\hline $\boldsymbol{*}$ & $\mathbf{0}$ & $\mathbf{1}$ & $\mathbf{2}$ & $\mathbf{3}$ \\
\hline $\mathbf{0}$ & 0 & 0 & 0 & 0 \\
$\mathbf{1}$ & 1 & 0 & 3 & 1 \\
$\mathbf{2}$ & 2 & 3 & 0 & 2 \\
$\mathbf{3}$ & 3 & 0 & 0 & 0 \\
\hline
\end{tabular}

Then $(X ; *, 0)$ is a proper BCL-algebra. It is easy to verify that there are

BCI-1: $((2 * 3) *(2 * 1)) *(1 * 3)=(2 * 3) * 1=2 * 1=3 \neq 0$. BCH-3: 1) On the left side of equation is $(2 * 3) * 1$ $=2 * 1=3$;

2) On the right side of equation is $(2 * 1) * 3=3 * 3$ $=0$.

In the expression we see that $3 \neq 0$. In fact, it is not difficult to verity that BCL-1, BCL-2 and BCL-3 are valid.

Example 2.1. The set $X=\{0,1, a, b\}$. Define a binary operation * on $X$ given by the following * multiplication table:

\begin{tabular}{ccccc}
\hline$*$ & $\mathbf{0}$ & $\mathbf{1}$ & $\boldsymbol{a}$ & $\boldsymbol{b}$ \\
\hline $\mathbf{0}$ & 0 & 0 & 0 & 0 \\
$\mathbf{1}$ & 1 & 0 & $b$ & 1 \\
$\boldsymbol{a}$ & $a$ & $b$ & 0 & $a$ \\
$\boldsymbol{b}$ & $b$ & $a$ & 0 & 0 \\
\hline
\end{tabular}

forms a proper BCL-algebra.

Example 2.2. Let $X=\{0,1,2,3\}$. We define a binary operation * on $X$ by 


\begin{tabular}{lllll}
\hline $\boldsymbol{*}$ & $\mathbf{0}$ & $\mathbf{1}$ & $\mathbf{2}$ & $\mathbf{3}$ \\
\hline $\mathbf{0}$ & 0 & 1 & 2 & 3 \\
$\mathbf{1}$ & 1 & 0 & 1 & 2 \\
$\mathbf{2}$ & 2 & 0 & 0 & 0 \\
$\mathbf{3}$ & 3 & 2 & 0 & 0 \\
\hline
\end{tabular}

It is not difficult to verify that $(X ; *, 0)$ is a proper BCL-algebra.

Fact 2.1. The example above implies BCL-algebra theory is independent algebra system.

Theorem 2.5. 1) A proper class is composed of all BCL-algebras; it is called a BCL-algebraic class;

2) All $\mathrm{BCK} / \mathrm{BCI} / \mathrm{BCH}$-algebraic class are proper subclass of BCL-algebraic class.

Proof: 1) By BCI / $\mathrm{BCH}$-algebraic class is a proper class, and by Theorem 2.3 tells us that BCL-algebraic class is a proper class.

2) By Theorem 2.4 it is obvious that $\mathrm{BCK} / \mathrm{BCI}$ $/ \mathrm{BCH}$-algebraic class is a proper subclass of $\mathrm{BCL}$-algebraic class.

Remark 2.1. It is not necessary that the algebra $\left(X ;^{*}, 0\right)$ of all type $(2,0)$ are BCL-algebras. Let's work out an example.

Example 2.3. Let $X=\{0,1,2\}$. Define a binary operation * on $X$ by

\begin{tabular}{llll}
\hline $\boldsymbol{*}$ & $\mathbf{0}$ & $\mathbf{1}$ & $\mathbf{2}$ \\
\hline $\mathbf{0}$ & 0 & 1 & 2 \\
$\mathbf{1}$ & 0 & 0 & 1 \\
$\mathbf{2}$ & 2 & 1 & 0 \\
\hline
\end{tabular}

Then $(X ; *, 0)$ is not BCL-algebra, we obtain

$$
(((1 * 2) * 1) *((1 * 1) * 2)) *((1 * 2) * 1)=2 * 0=2 \neq 0 .
$$

BCL-3 does not hold for the algebra of type $(2,0)$. But $1 * 1=0$, it is satisfies BCL- 1 and BCL-2.

Theorem 2.6. Let $\left(X ;{ }^{*}, 0\right)$ be a BCL-algebra, we have the following relations: for any $x, y, z \in X$,

1) BCI-2: $(x *(x * y)) * y=0$;

2) BCI-5: $x^{*} 0=0$ imply $x=0$.

Proof: The theorem follows immediately from Defini- tion 1.1 above.

Theorem 2.7. A BCL-algebra $(X ; *, 0)$ is called proper BCL-algebra if and only if it not satisfies BCI-1 and $\mathrm{BCH}-3$.

Proof: Necessity. If $(X ; *, 0)$ satisfies $\mathrm{BCI}-1$ and BCH-3. By Theorem 2.6, it satisfies BCI-2 and BCI-5, and then we obtain a $\mathrm{BCI}$-algebra and a $\mathrm{BCH}$-algebra, contradicting conditions of a proper BCL-algebra.

Sufficiency. Since $(X ; *, 0)$ not satisfies BCI-1 and BCH-3. Therefore, it is not BCI-algebra and $\mathrm{BCH}$-algebra but proper BCL-algebra.

Theorem 2.8. Algebra $(X ; *, 0)$ of type $(2,0)$ is a BCL-algebra if and only if it satisfies the following conditions: for all $x, y, z \in X$,

1) BCL-1: $\quad x^{*} x=0$;

2) BCL-2: $x * y=0$ and $y^{*} x=0$ imply $x=y$;

3) $((x * y) * z) *\left(\left(x^{*} z\right) * y\right)=(z * y) * x$.

Proof: We shall only prove the Theorem 2.8 3), by Theorem 2.1, Theorem 2.3 and Definition 1.2. So 3) is valid.

\section{Conclusions}

Taking theory of sets and propositional calculus as the backdrop, the new study suggests that the BCL-algebras are an important algebra in the axiom algebra system, which delves into generalizations of difference operations and characteristic.

\section{References}

[1] Y. Imai and K. Iséki, "On Axiom System of Propositional Calculi XIV," Proceedings of the Japan Academy, Vol. 42, No. 1, 1966, pp. 19-22. doi:10.3792/pja/1195522169

[2] K. Iséki, "An Algebra Related with a Propositional Calculus," Proceedings of the Japan Academy, Vol. 42, No. 1, 1966, pp. 26-29. doi:10.3792/pja/1195522171

[3] K. Iseki, "On BCI-Algebras," Mathematics Seminar Notes (Kobe University), Vol. 8, No. 1, 1980, pp. 125-130.

[4] Q. P. Hu and X. Li, "On BCH-Algebras," Mathematics Seminar Notes (Kobe University), Vol. 11, No. 2, 1983, pp. 313-320.

[5] Q. P. Hu and X. Li, "On Proper BCH-Algebras," Mathematica Japonica, Vol. 30, No.4, 1985, pp. 659-661.

[6] Y. S. Huang, "BCI-Algebra," Science Press, Beijing, 2006, p. 21. 